\title{
Brillouin Frequency Shift Sensing Technology Used in Railway Strain and Temperature Measurement
}

\author{
Wen-Kuei Hsu ${ }^{1, *}$, Yu-Lin Lee ${ }^{2}\left(\mathbb{D}\right.$ and Tsan-Teng Kuan ${ }^{2}$ \\ 1 Department of Civil and Disaster Prevention Engineering, National United University, No. 2 Lienda Rd., \\ Miaoli 36003, Taiwan \\ 2 Department of Civil Engineering, Chung Hua University, No. 707, Sec.2, Wufu Rd., Hsinchu 30012, Taiwan; \\ rosalee@chu.edu.tw (Y.-L.L.); mttkuan2@gmail.com (T.-T.K.) \\ * Correspondence: hsuwen918@gmail.com; Tel.: +886-905-585-918
}

Citation: Hsu, W.-K.; Lee, Y.-L.;

Kuan, T.-T. Brillouin Frequency Shift Sensing Technology Used in Railway Strain and Temperature

Measurement. Appl. Sci. 2021, 11,

7101. https://doi.org/10.3390/

app11157101

Academic Editor: Nicola Bosso

Received: 22 June 2021

Accepted: 27 July 2021

Published: 31 July 2021

Publisher's Note: MDPI stays neutral with regard to jurisdictional claims in published maps and institutional affiliations.

Copyright: (c) 2021 by the authors. Licensee MDPI, Basel, Switzerland. This article is an open access article distributed under the terms and conditions of the Creative Commons Attribution (CC BY) license (https:// creativecommons.org/licenses/by/ $4.0 /)$.

\begin{abstract}
This study and verification are based on the Brillouin frequency shift (BFS), which is related to the strain and temperature changes of a single-mode fiber, because such a shifted frequency can be quantitatively measured and converted to strain and temperature differences. We explain the installation of a Brillouin distributed fiber sensing system (DFOS) on an actual operating railway to measure the temperature and strain of the rail. In addition, the measured data were calculated and analyzed, revealing the geometric irregularity of the tested rail and the location of the abnormality. We obtained a temperature difference of $12.1^{\circ} \mathrm{C}$ between the temperature distribution of the measured rail and the atmospheric temperature, and there was a $1.5 \mathrm{~h}$ delay between the two. We also obtained rail irregularities ranging from -0.3 to $+0.4 \mathrm{~mm}$ by calculating the slight strain difference of the rail in this test.
\end{abstract}

Keywords: Brillouin frequency shift; distributed fiber optic sensing; rail strain; rail deformation; rail irregularity

\section{Introduction}

The safety of railway operation mainly depends on whether the rail and roadbed are abnormal. When an abnormality occurs, can we effectively respond to the abnormal status and location and then take necessary action to prevent further disasters? This is one of the most important management issues. At present, in the Taiwan Railway Administration Bureau, rail inspection mostly depends on the personnel's visual inspection and off-peak inspection vehicles, both of which cannot immediately respond to abnormal conditions and locations; therefore, these personnel cannot make effective management decisions. An effective, precise, and automatic monitoring technology is urgently needed.

Brillouin distributed optical fiber sensing technology can measure the strain and temperature changes of single-mode optical fibers. In this research, we applied the technology to railway temperature and strain measurements, with reference to [1], which discussed the use of an extra positioning clamp, resulting in noncontinuous data points. Due to a steel rail being a continuous object, any location can be a point of breakage. Through experimentation, an adhesion method was used in this research to increase data sampling density to obtain continuous measurement data and increase positional accuracy. In addition, we were able to transfer data through the internet to a computing server and display the results of strain in graphical and numeral values on-screen, which can provide personal data for setting managing values and plans of action. To further enhance the monitoring function with instant feedback, when measurements exceed alarm levels, the system automatically notifies related personnel to intervene. This paper describes and explains the results of our installation, configuration, measurement, and analysis. 


\section{Measurement Theory}

\subsection{Brillouin Back-Scattering in Silica Single-Mode Fiber}

When incident light is transmitted through an optical fiber, the light passes through the silica molecules and other doping particles in the optical fiber. If the radius of these particles is smaller than the wavelength of the incident light, light scatters in all spatial directions. Back-scattering light specifically refers to scattered light in the opposite direction to the incident.

There are three types of scattering: Rayleigh scattering, Raman scattering, and Brillouin scattering. The frequency spectrum in Figure 1 [2] shows the scattering and frequency band that correspond to $1550 \mathrm{~nm}$ incident light.

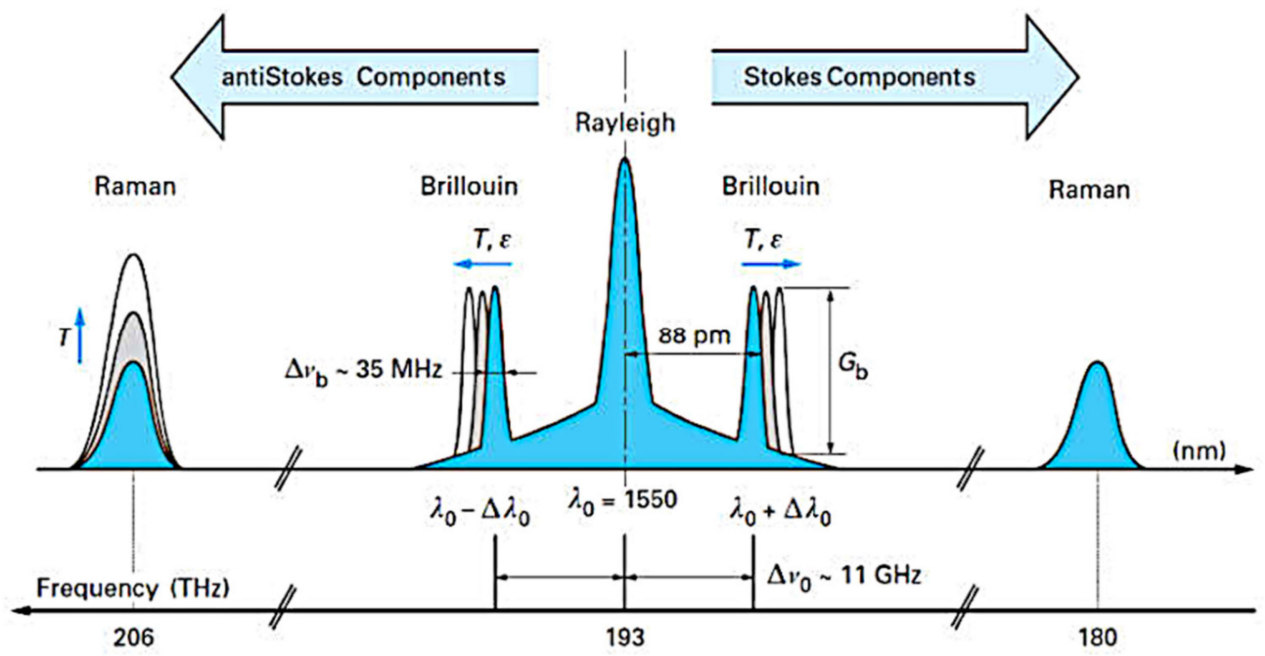

Figure 1. The back-scattering in silica single-mode fiber: there are three (3) scattering frequencies in single-mode fiber against the $1550 \mathrm{~nm}$ incident light (following the work of Ferdinand, 2014). Note that the Brillouin scattering frequency is only compounded by temperature $(\mathrm{T})$ and strain $(\varepsilon)$.

Brillouin scattering refers specifically to inelastic scattered light, and it is mainly caused when phonons (acoustic phonons) collide with incident light and generate scattering with a lower power and different frequencies. From this phenomenon, we can gather the distance of an event in the fiber by counting the time difference between the incident light and back-scattered light. In the meantime, by measuring the frequency difference of the back-scattered light and incident light, we can obtain the values of the external strain and temperature that affected the fiber.

\subsection{Brillouin Frequency Shift}

Since Brillouin scattering is inelastic scattering caused by the collision of incident light and scattered phonons, the frequencies generated at the Stokes and anti-Stokes zones are different from the original frequency of the incident, the frequencies of which comprise the Brillouin frequency shift (BFS); this can be expressed as Equation (1):

$$
v_{B}=2 n V_{a} / \lambda
$$

where $n$ is the refractive index of the optical fiber, $V_{a}$ is the acoustic wave velocity in the optical fiber, and $\lambda$ is the wavelength of incident light.

Horiguchi et al., 1995, calculated the BFS center frequency [3]. They used $n=1.46$, $V_{a}=5945 \mathrm{~m} / \mathrm{s}$, and $\lambda=1.32 \mu \mathrm{m}$ as the calculation parameters of a standard single-mode fiber. The BFS center frequency outcome was $13.15 \mathrm{GHz}$. In this test, a pumping light with a $1.55 \mu \mathrm{m}$ wavelength was used, so we obtained a BFS center frequency of $11.2 \mathrm{GHz}$.

The research by Horiguchi et al., 1989 and Kurashima et al., 1990 showed that the Brillouin frequency shift is highly related to the external strain [4] and temperature [5] 
acting on a fiber, and the BFS can be quantitatively analyzed based on its linear parameters. Hence, the relationship between Brillouin frequency shift, strain, and temperature is shown in Equation (2):

$$
\begin{gathered}
v_{B}=v_{B 0}+C_{\varepsilon}\left(\varepsilon-\varepsilon_{0}\right)+C_{T}\left(T-T_{0}\right) \\
\Delta v_{B}=C_{\varepsilon} \Delta \varepsilon+C_{T} \Delta T
\end{gathered}
$$

where $v_{B}$ is the measured Brillouin frequency shift, which combines two factors of strain, $(\varepsilon)$ and temperature $(T) ; v_{B 0}$ is the initial value of the Brillouin frequency shift at a specific temperature; and $C_{\varepsilon}$ and $C_{T}$ are the coefficients of strain and temperature, respectively. Here, both $C_{\varepsilon}$ and $C_{T}$ were related to the characteristics of the outer package of the optical fiber; actual coefficients are specific to each fiber manufacturer.

Schenato, 2017, found $C_{\varepsilon}$ and $C_{T}$ reference values of $0.046 \mathrm{MHz} / \mu \varepsilon$ and $1.07 \mathrm{MHz} /{ }^{\circ} \mathrm{C}$, respectively [6]. Therefore, it can be stated that the difference of the initial and measured BFS values is the sum of the strain and temperature differences.

In this experiment, $C_{\varepsilon}$ and $C_{T}$ were $0.0497 \mathrm{MHz} / \mu \varepsilon$ and $1.07 \mathrm{MHz} /{ }^{\circ} \mathrm{C}$, respectively; the constants were given by the optic cable supplier Neubrex [7] for the FN-SSL-3 model cable.

The above mentioned BFS sensing method had been used in railway composite bogies [8] and railway bridge [9] measurement.

\section{Methods of Test and Verification}

\subsection{Outline of the Test Field}

For this test, we chose a portion of railway that is an operational line in Pingtung, Taiwan, as the test field. The outline is shown as Figure 2. The adhesion locations (D, E, and F) of the measuring optical cable are shown in Figure 3. Figure 4 shows the cable after adhesion. The set-up of the reference rail is shown in Figure 5. A schematic of the data collection and internet communication structure is shown in Figure 6.

\section{Pingtung station}

\section{Guilai station $\Rightarrow$}

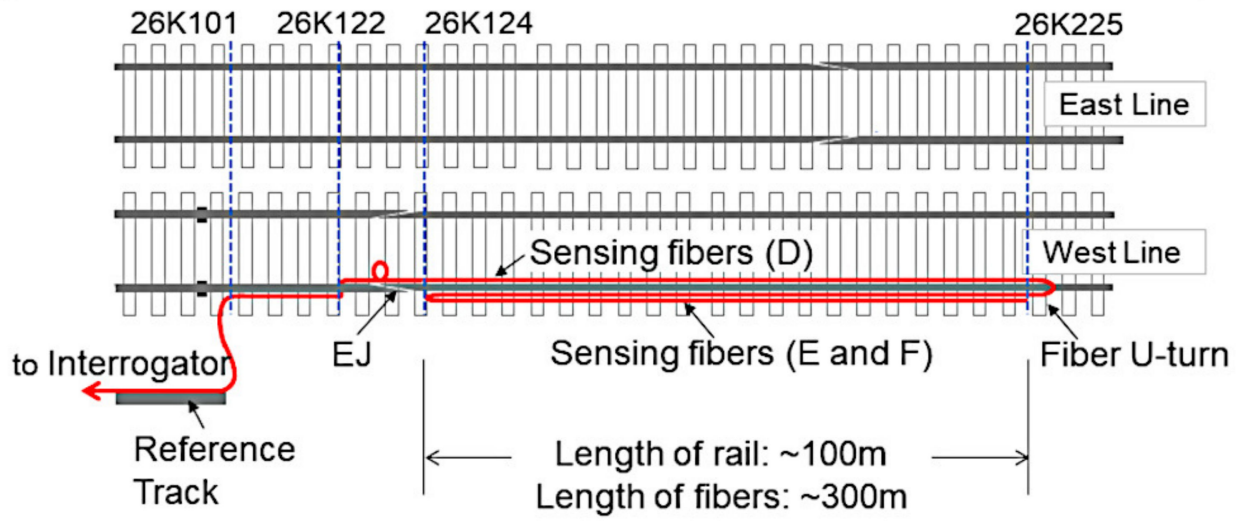

Figure 2. The sensing fiber deployment diagram: the location and length of the tested rail, reference track, and three (3) installed segments of sensing fibers. The sensing fibers were fuse-spliced into one (1) test path.

\subsection{Description of the Outline}

We installed a distributed fiber optic sensing system (DFOS) in Pingtung Rail Station that was $2 \mathrm{~km}$ away from the tested rail (not shown in Figure 2). The DFOS contains a commercial Brillouin optical time domain interrogator, and its measurement parameters were set as: a spatial resolution of $50 \mathrm{~cm}$, a measurement accuracy of $7 \mu \varepsilon$, a repeatability of $3 \mu \varepsilon$, and a test speed of $60 \mathrm{~s}$. In addition, the DFOS has a computer responsible for numerical calculations, a computer responsible for large amounts of test data storage and management, and an operating platform for presenting measurement results.

The test section is a standard $60 \mathrm{~kg} / \mathrm{m}$ ballast track. The tested rail starts at the expansion joint (EJ) and extends $100 \mathrm{~m}$ in the direction of increasing length. It belongs 
to the movable section of the continuous welded rail (CWR) and covers all accessories of track engineering, such as the fish plate, welding points, and fasteners.

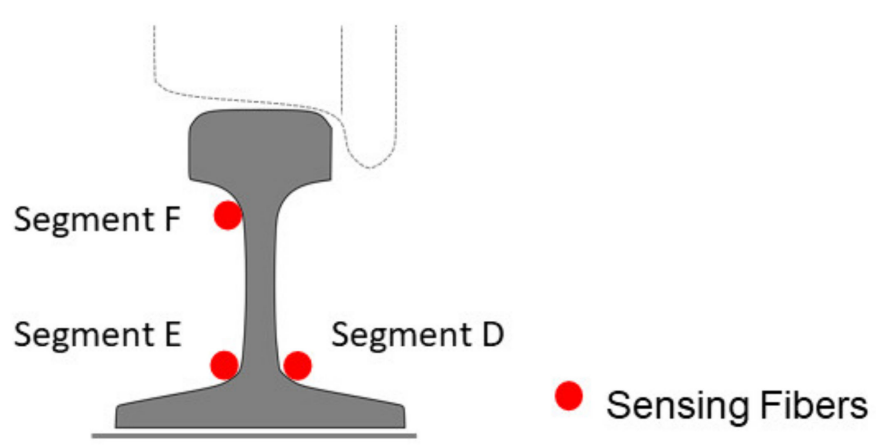

Figure 3. A cross-section view of the tested rail: the installed locations of fibers and the related positions.

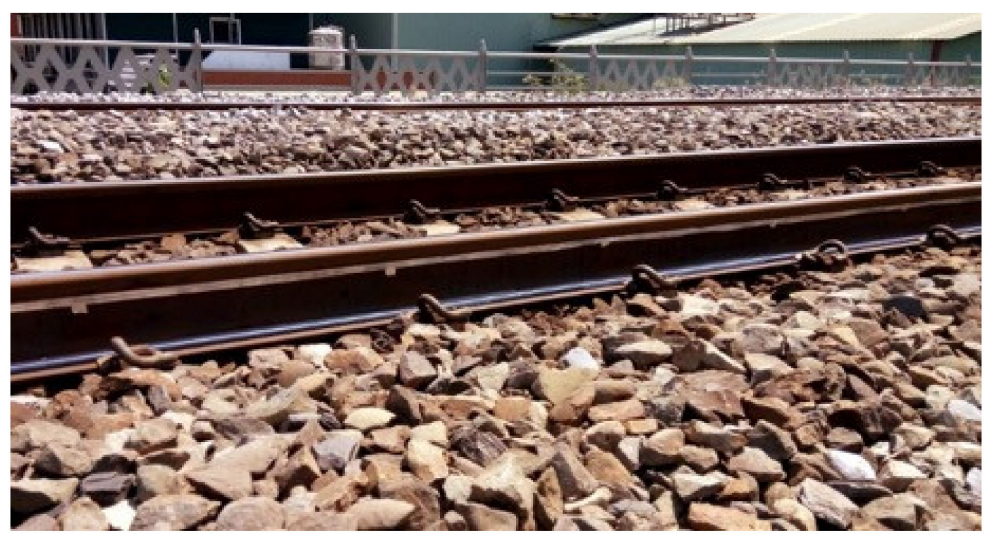

Figure 4. The sensing fibers after the installation: this picture was taken at mile $26 \mathrm{~K} 125$ in the test field. To protect against fiber breakage from the impact of ballasts, all sensing fibers were covered by aluminum tape.

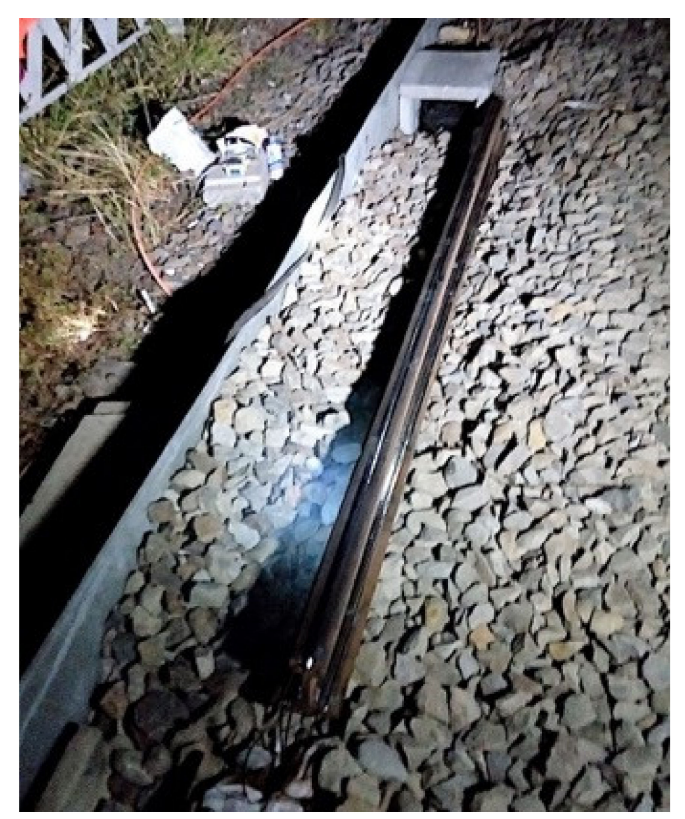

Figure 5. The reference track: this reference track was placed at mile $26 \mathrm{~K} 101$ and was kept $2 \mathrm{~m}$ away from and parallel to the tested rail in the field. 


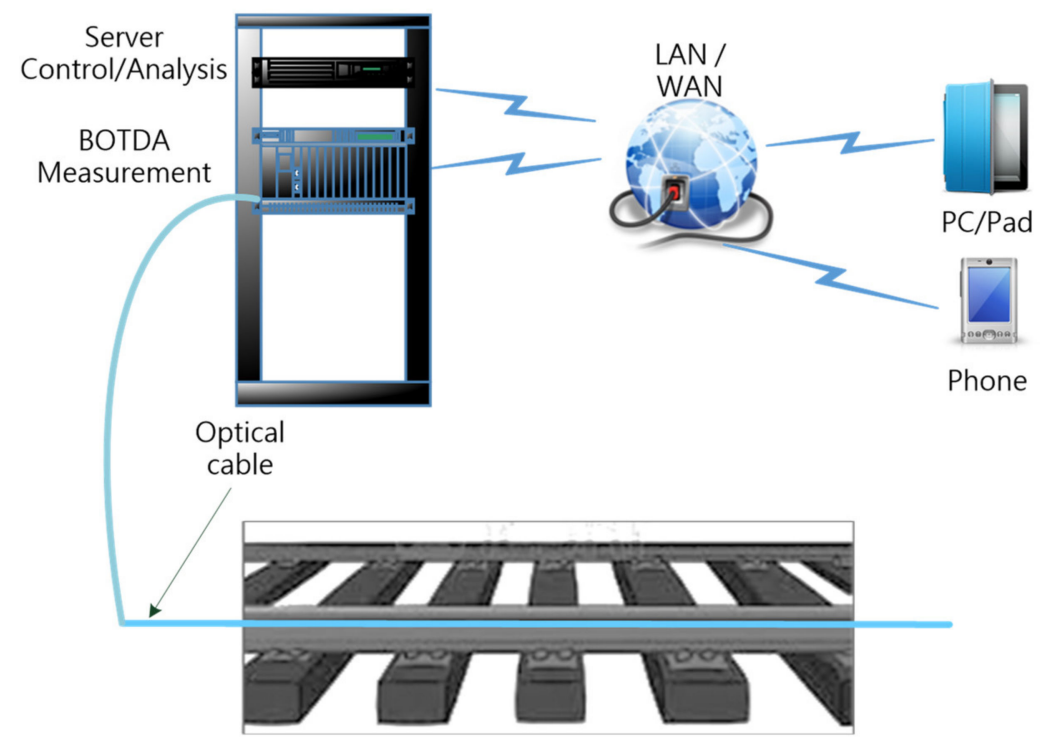

Figure 6. Data collection and internet communication structure.

The strain measured from the sensing fiber was mainly from the direction of its horizontal axis, such as with the expansion of rail, but the external force was mainly from vertical axis, such as with the weight and rolling of train. It was necessary to attach sensing fibers to three spatial positions of the rail, which were the main data sources for our strain measurement and displacement analysis.

In this test, we attached sensing fibers at the left foot, right foot, and upper web of the tested rail. In addition to the measured strain in the long axis direction of the rail, the displacement in the horizontal and vertical directions was calculated based on the magnitude of the strain that was measured by each individual fiber and their symmetrical difference in stress. The fiber attachment positions are shown in Figures 3 and 4.

We set the measurement reference track parallel to the side of mile 26K101 and kept it $2 \mathrm{~m}$ from the tested rail. The reference track and the tested rail were at the same ambient temperature and had the same coefficient of thermal expansion, so they can be regarded as having the same temperature. We placed three sensing fibers on the reference rail in parallel to those of the tested rail; the setting of the reference track is shown in Figure 5.

As shown in Figure 6, the system was separated into two main functions. The first is measurement, where the server sends command through LAN/WAN to BOTDA to start measuring. After recording the measurement, BOTDA sends the measured data back to the server for analysis and saves them in the database. If the calculated values exceed the alarm level, messages are automatically sent to users with LAN/WAN. The second function is the presentation of results. Users can use a PC/pad/phone to obtain the latest measurement results, which are graphically shown on the device.

\subsection{Compensation of Rail Thermal Expansion}

To calculate strain, the temperature distribution of the rail must also be considered. Temperature change is mainly derived from solar thermal radiation and atmospheric temperature. A steel rail is considered to be a high thermal conductivity material, so it was assumed that the three differently-located fibers on the rail experienced the same thermal conditions. Therefore, in the marginal time of measurement, the overall temperature measured by the three sensing fibers could still be regarded as the same. There was no distinction in temperature measured even though the neutral axisymmetric difference of the cross-section of rail was I-shaped.

However, because the section of the tested rail is within the movable section of the continuous welded rail, if the Brillouin frequency measured by the tested rail was directly used to calculate the temperature and strain values, errors may have occurred due to 
ignoring the variation in the thermal expansion of the rail. We added an affecting factor of thermal expansion of the rail to the equation, as shown in Equation (3):

$$
\Delta v_{B}=C_{\varepsilon} \Delta \varepsilon+C_{\varepsilon}(\alpha \times \Delta T)+C_{T} \Delta T
$$

where $\alpha$ is thermal expansion coefficient of the rail.

\subsection{The Separation of Temperature and Strain}

The measured Brillouin frequency shift was a combination of two variables: the strain $(\varepsilon)$ and temperature $(T)$. To obtain the strain of the tested rail, we tried to obtain its temperature measurement first.

The reference track was set without fasteners or sleepers so that it could be freely retractable without being restricted by external forces. There was no strain difference between the optical fibers on the reference and tested tracks $(\Delta \varepsilon=0)$, and the temperature of the reference track can be calculated using Equation (4):

$$
\begin{gathered}
\Delta v_{B}=C_{\varepsilon}(\alpha \times \Delta T)+C_{T} \Delta T \\
\Delta T=\Delta v_{B} /\left(C_{\varepsilon} \times \alpha+C_{T}\right) \\
T=T_{r e f}+\Delta v_{B} /\left(C_{\varepsilon} \times \alpha+C_{T}\right)
\end{gathered}
$$

where $T_{\text {Ref }}$ is the initial temperature measured when the reference track was installed.

Since the reference track and the tested rail were in the same ambient temperature conditions, the specifications of the reference track were the same as those of the tested rail and had the same thermal expansion coefficient, so the temperature measured from reference track was considered to be the same as the temperature of the tested rail. The measured temperature curve is shown in Figure 7.

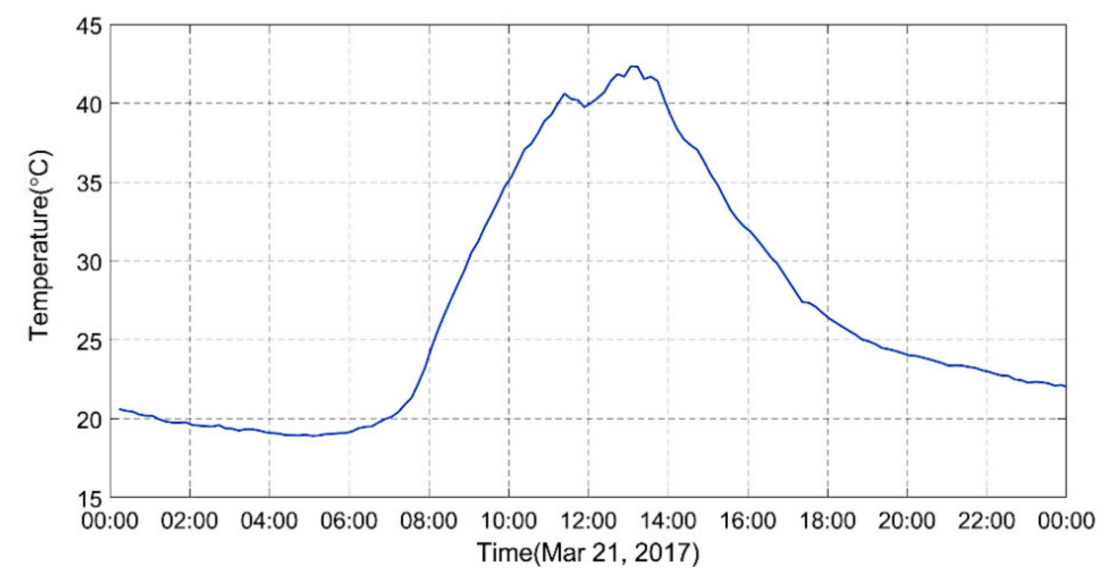

Figure 7. The temperature profile of the reference track: the temperature measured from the reference track, which was also used as the temperature of the tested rail.

\section{Measurement and Calculation Results}

\subsection{The Temperature Distribution and Changes over Time}

Though the temperature curves of traditional measurements can only represent single locations, Figure 8 shows the temperature changes of the tested rail under continuous monitoring for $48 \mathrm{~h}$, as well as the distributed temperature according to the entire $100 \mathrm{~m}$ length rail. The coordinates of Figure 8 use temperature value, time, and length for its three-axis analysis graph, showing the temperature changes of any rail position at different times. 


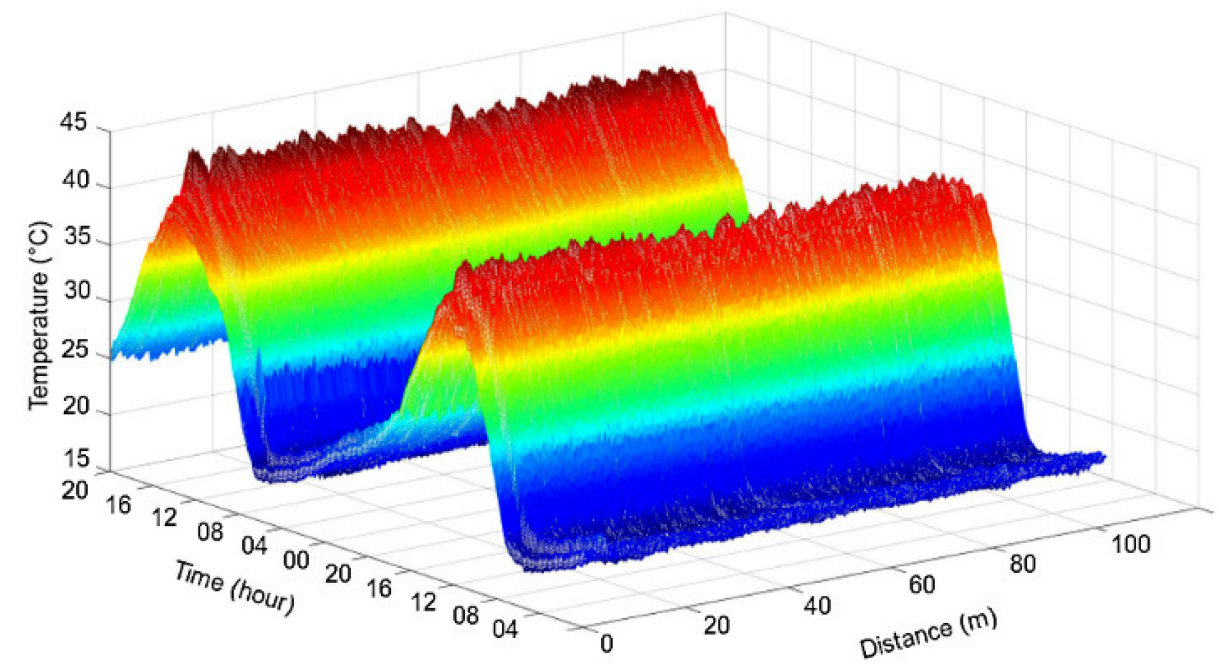

Figure 8. The 3D measured temperature profile: the temperature distribution of the tested rail in the 3 dimensions of time, distance, and temperature, which is quite different from the temperature profiles of other single-point measurement results.

We downloaded weather observation data from the C0R170 weather station [10] on the same day as the field test for a comparison with measurement data. The results are shown in Figure 9. The rail and atmospheric temperature rose and fell with the same trend.

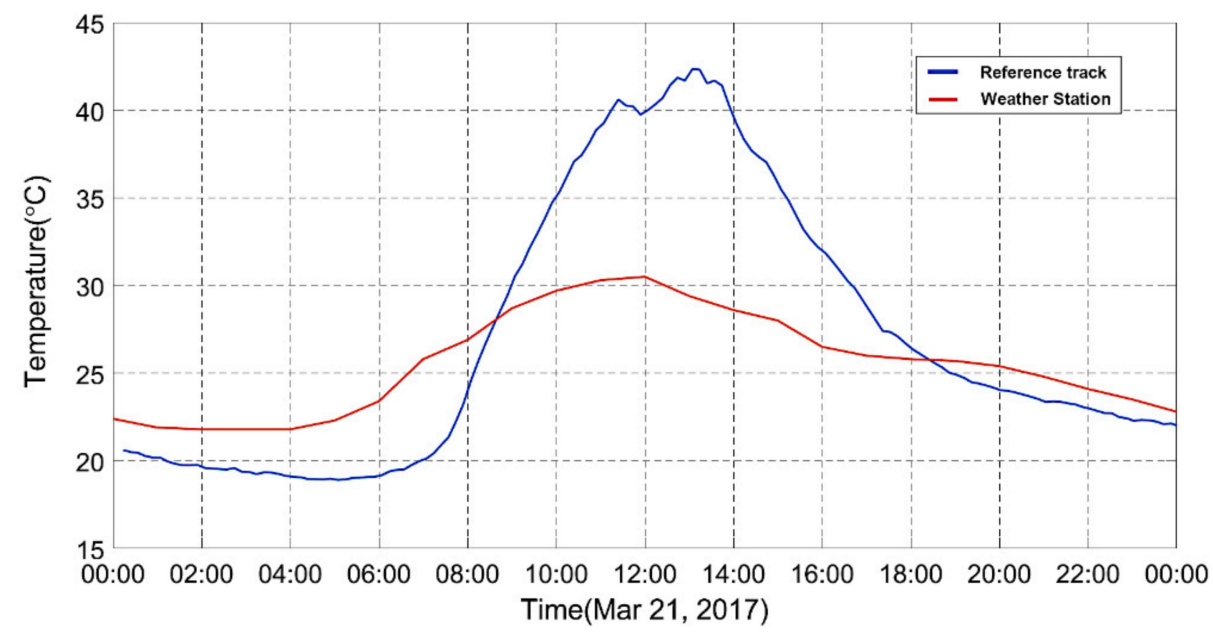

Figure 9. A temperature comparison of rail and atmospheric temperature.

Both the heat radiation under sunshine in the daytime and the specific heat capacity of the rail being larger than that of air caused the rail temperature to be $12.1^{\circ} \mathrm{C}$ higher than the atmospheric temperature, and there was a lag time difference of about $1.5 \mathrm{~h}$. In addition, the lowest temperature of the rail was about $3.0^{\circ} \mathrm{C}$ lower than the atmospheric temperature due to rainfall and radiation cooling effects in the early morning, e.g., 5:30 a.m. For inspectors who are concerned about the extension characteristics of rails, this measurement result can be used as a reference for determining the inspection time when performing personnel inspection tasks.

\subsection{The Strain Distribution and Changes over Time}

Figure 10 shows the intensity of the strain distributed over the tested rail in the $24 \mathrm{~h}$ test period. The calculation was based on the BFS of the reference track in Equation (5):

$$
\Delta \varepsilon=\left(\Delta v_{B_{\text {test }}}-\Delta v_{B_{r e f}}\right) / C_{\varepsilon}
$$


where $\Delta v_{B_{\text {test }}}$ is the BFS of the tested rail and $\Delta v_{B_{\text {ref }}}$ is the BFS of the reference track.

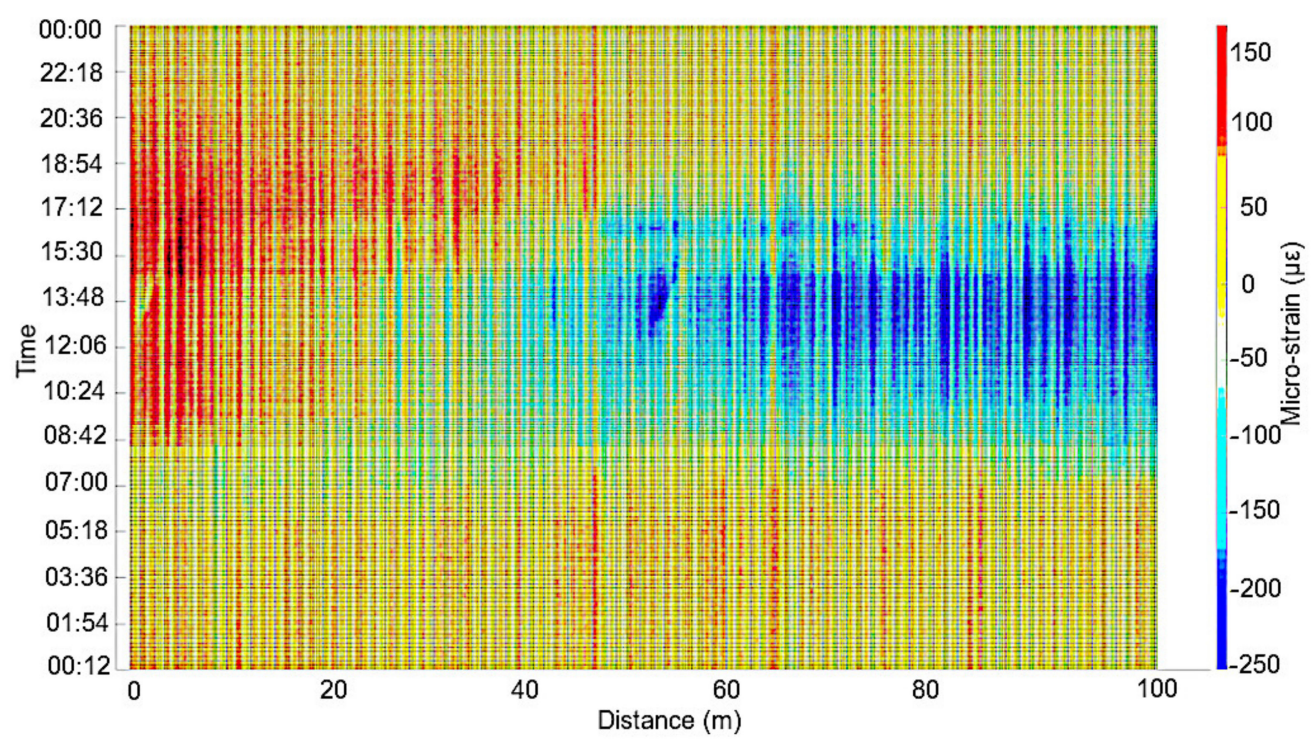

Figure 10. The strain distribution profile: the strain distribution varies because the restraining force is different in the unmovable area (on the right) and the movable area (on the left).

The tested rail was found to have less free stretch than the reference track due to the restrictions of sleepers and ballasts.

As shown in the figure, the strain near the unmovable section was negative, as shown on the right of the figure, which means the expansion was less than that of the reference track. On the left side of the figure, the strain in the movable section is positive. It can be understood that because greater restraining force was from sleepers and ballasts in the unmovable section, an axial strain that was even smaller than that of the freely extending reference track was measured. In contrast, farther away from the unmovable section, a larger amount of strain was measured, and the maximum strain was reached at the extension joint (EJ) at the end of rail, as shown at distance zero (0) on the left of the figure.

In Figure 10, regular vertical dark stripes appear along the direction of increasing length. A total of 167 stripes were calculated and are shown. Taking the sensing fiber segment D as $101.45 \mathrm{~m}$, the stripe interval was calculated to be $0.61 \mathrm{~m}$, which roughly coincides with the average distance between the sleepers in the verification area.

\subsection{The Displacement Distribution and Changes over Time}

Based on the measurements of the distributed fiber optic sensing system that was used in this test, Figure 11 shows the strain data of the tested rail. The four areas of A, $\mathrm{B}, \mathrm{C}$, and D sustained strain, indicating that these places are more abnormal than others. The picture shows that the steel rail repeatedly experienced similar strain. The railway experienced greater strain at times where temperatures were higher (09:20 14:50) and lower strain when temperatures were lower. This explains the accumulation and release of the stress of the steel rail. The areas A, B, C, and D experienced high strain for long periods of time. Relevant railroad personnel should examine these abnormal spots. 


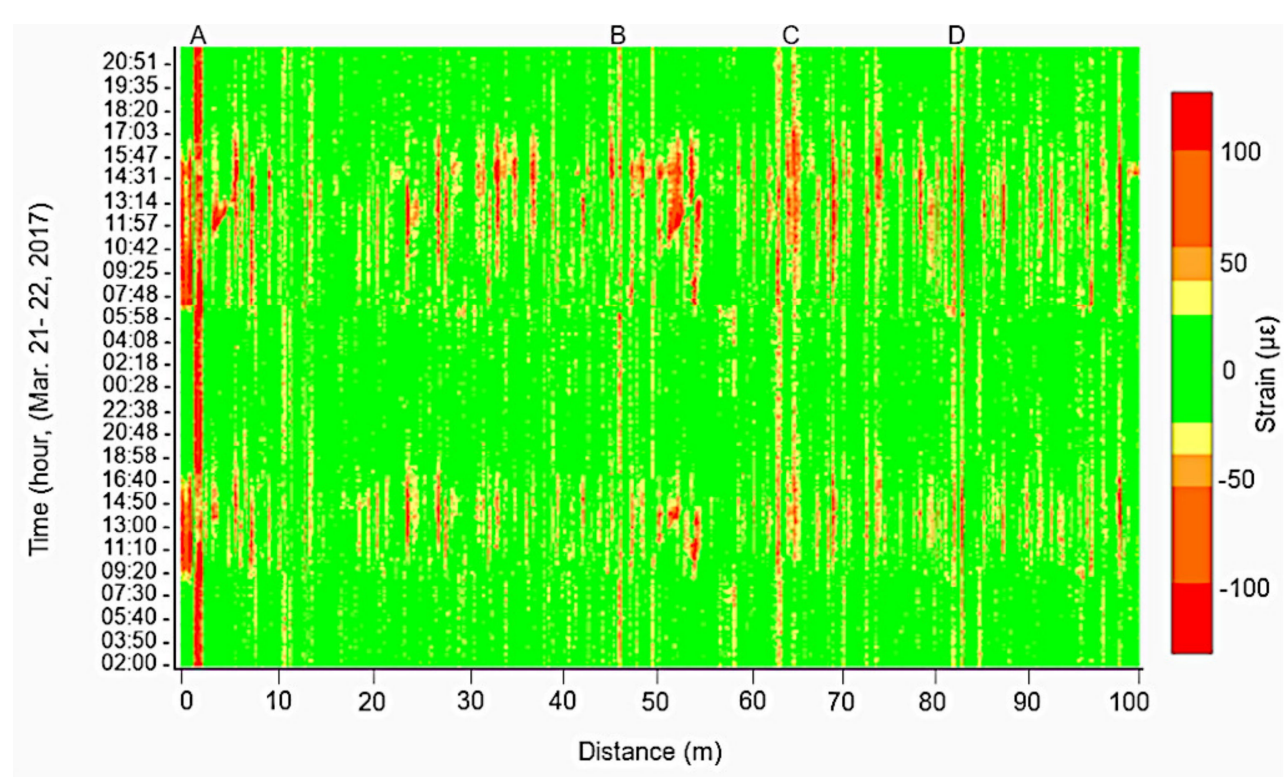

Figure 11. The $48 \mathrm{~h}$ strain distribution: the strain distribution of the tested rail over a $48 \mathrm{~h}$ period, showing four abnormal areas of A, B, C and D.

In addition, with the large data calculation and combined analysis capabilities of the sensing system, we were able to calculate the measured strain difference $(\Delta \varepsilon)$ in units of $0.5 \mathrm{~m}$. Displacement was calculated by integrating the strain shown in Equation (6). Then, the simple rectangular rule was used to estimate values through Expression (7):

$$
\begin{gathered}
D_{x}=\int \varepsilon_{x} d x+D_{0} \\
D_{x}=0.5 \cdot \sum \varepsilon_{x}+D_{0}
\end{gathered}
$$

where $D_{x}$ is the displacement and $\varepsilon_{x}$ is the strain difference at distance $x$. Displacement already existed in the railway before installing the fiber. We focused on measuring the displacement change after installation, so there was no need to consider the previous displacement of the rail. In the calculation, the initial value of displacement was equal to zero, $D_{0}=0$. Furthermore, the tested rail displacement was measured to be from -0.3 to $+0.4 \mathrm{~mm}$, as shown in Figure 12 .

Through the calculation of strain distribution data from Figure 11, we created Figure 12, which is easier to comprehend. This displacement distribution can be provided to railway management to assess abnormal railway data. In Figure 12, there are some significant regions-both the stretched (red) and compressed (blue) regions- that are different from other regions with zero displacement.

We tried to study these abnormal regions in the context of the overall structure of the track. We know that the main function of the ballast and track bed is to maintain rail elasticity, disperse train loads, and absorb driving shock, but the measured results of our study showed that the rail's recovery elasticity is insufficient. In addition, our chosen verification field was a section where the operational line was recently converted from ground into an elevated section; during the verification days, the track adjustment and ballast compaction were still in progress at night. Therefore, we speculate that the abnormal displacement of the rail may have been due to the uncompleted compaction of the ballast or track slab. However, this inference needs further research and verification. 


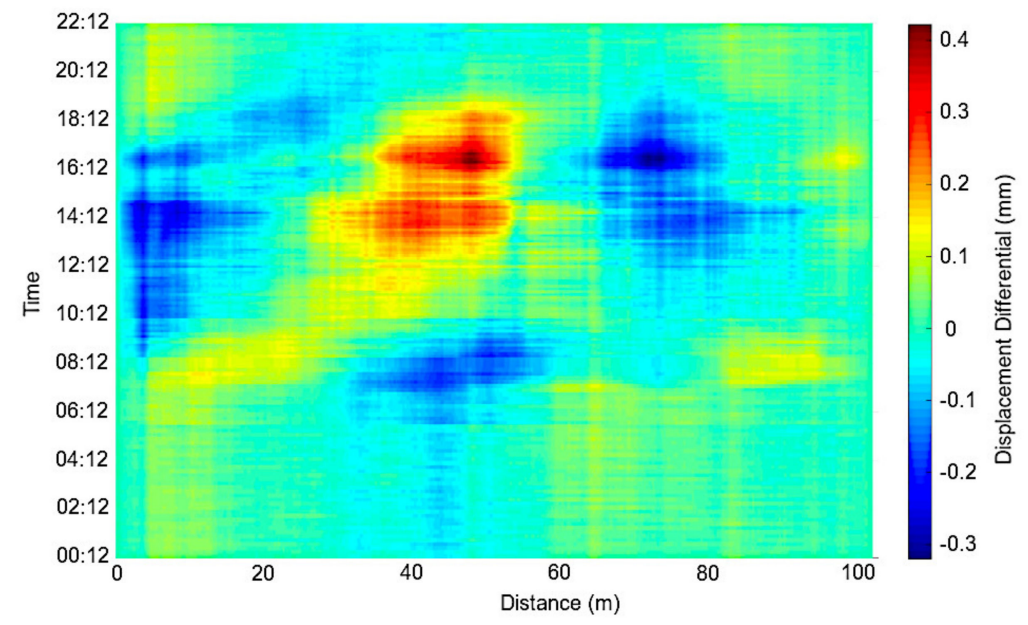

Figure 12. Suspect location of displacement: Displacement distribution derived from the strain differential $(\Delta \varepsilon)$ of the rail. The displacement values in colored places are worth noting.

\section{Conclusions}

In contrast to tests using weather stations or visual inspection, the test used in this study was found to be able to clearly identify various temperatures of a rail. We obtained a temperature difference of $12.1^{\circ} \mathrm{C}$ between the temperature distribution of the measured rail and the atmospheric temperature, and there was $1.5 \mathrm{~h}$ delay between the two.

In addition, we obtained rail irregularities ranging from -0.3 to $+0.4 \mathrm{~mm}$ by calculating the slight strain difference of the rail in this test. We were also able to distinguish the unmovable and movable zones to derive the numbers and spacing of the sleepers. Ultimately, we calculated the position of the abnormal area and graphically presented it on the screen of the sensing system.

This test is performed by a DFOS. The temperature and strain changes can be measured with the fiber Brillouin frequency shift, and then one can obtain temperature, strain and rail displacement values based on a reference track. By observing the strain, displacement, and temperature distribution of a rail across time, one can determine whether a railroad is abnormal.

We reached several useful conclusions, including the following: (1) at any time, the temperature distribution and continuous change of a main rail can be measured with this test; (2) this test can accurately measure the geometric irregularities, displacement distribution, and abnormal positions of a rail; and (3) after setting the warning and action values of critical parameters, test data and warning messages can be automatically displayed with graphical charts.

Using the above-tested foundation, we will continue to conduct relevant research and verification regarding track characteristic issues, and we will continue to improve system accuracy and efficiency. Future research directions include: (1) measuring the stress accumulation and release of continuous welded rails, as well as comparing the effects of temperature on this phenomenon; (2) testing the strain and temperature distribution of continuous welded rails, especially in curved sections, and verifying the displacement relationship; and (3) analyzing and comparing DFOS sensing results with actual maintenance engineering for a better system.

This is the first DFOS test for the direct measurement of an operating main line in the Taiwan Railway. We successfully translated measurement results into graphical outcomes that are more convenient for maintenance and management teams to operate and judge. Additionally, we hope that the distributed optical fiber sensing technology can promote the benefits of track maintenance. 
Author Contributions: W.-K.H.: analysis, validation, and main editing and reviewing; Y.-L.L.: software programming and computation; T.-T.K.: on-site measurement and data curation. All authors have read and agreed to the published version of the manuscript.

Funding: This research received no external funding.

Institutional Review Board Statement: Not applicable.

Informed Consent Statement: Not applicable.

Data Availability Statement: Not applicable.

Acknowledgments: This test and study were conducted using the DFOS and sensing fibers that were provided by OlitGlobal Technology (Kaohsiung, Taiwan) to measure the strain and temperature of the tested rail. We also thank the Kaohsiung Branch of Taiwan Railway Administration and the Southern Office of Railway Reconstruction Bureau for their assistance during the test period.

Conflicts of Interest: The authors declare no conflict of interest. The sponsors had no role in the design, execution, interpretation, or writing of the study.

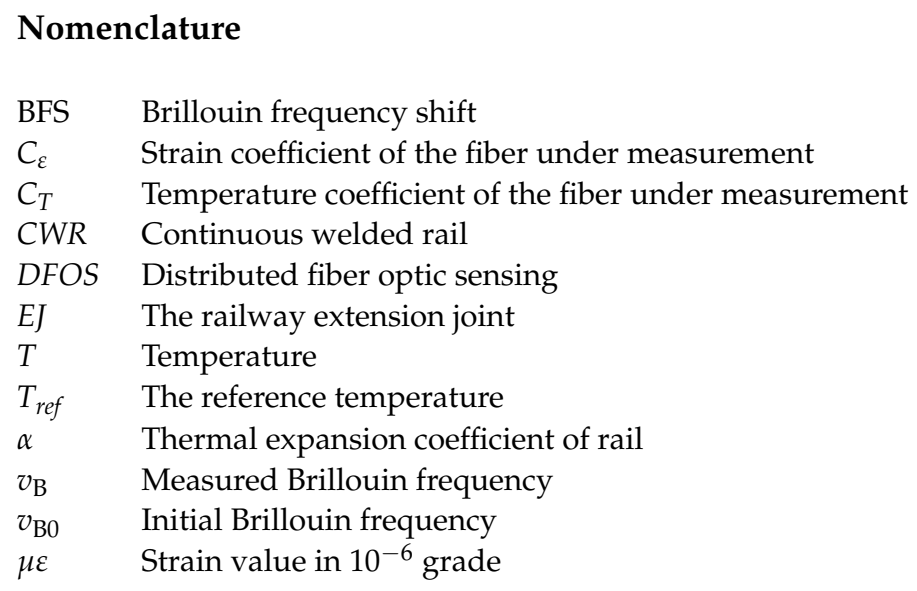

\section{References}

1. Klug, F.; Lackner, S.; Lienhart, W. Monitoring of railway deformations using distributed fiber optic sensors. In Proceedings of the Joint International Symposium on Deformation Monitoring (JISDM), Wien, Austria, 30 March-1 April 2016.

2. Ferdinand, P. The evolution of optical fiber sensors technologies during the 35 last years and their applications in structural health monitoring. In Proceedings of the EWSHM-7th European Workshop on Structural Health Monitoring, Nantes, France, 8-11 July 2014; Université de Nantes: Nantes, France, 2014; pp. 914-929.

3. Horiguchi, T.; Kurashima, T.; Tateda, M. Tensile strain dependence of Brillouin frequency shift in silica optical fibers. IEEE Photonics Technol. Lett. 1989, 1, 107-108. [CrossRef]

4. Horiguchi, T.; Shimizu, K.; Kurashima, T.; Tateda, M.; Koyamada, Y. Development of a distributed sensing technique using Brillouin scattering. J. Lightwave Technol. 1995, 13, 1296-1302. [CrossRef]

5. Kurashima, T.; Horiguchi, T.; Tateda, M. Thermal effects on the Brillouin frequency shift in jacketed optical silica fibers. Appl. Opt. 1990, 29, 2219-2222. [CrossRef] [PubMed]

6. Schenato, L. A review of distributed fibre optic sensors for geo-hydrological applications. Appl. Sci. 2017, 7, 896. [CrossRef]

7. Kishida, K.; Yamauchi, Y.; Guzik, A. Study of Optical Fibers Strain-Temperature Sensitivities Using Hybrid Brillouin-Rayleigh System. Photonic Sens. 2014, 4, 1-11. [CrossRef]

8. Yoon, H.; Kim, J.; Song, K.; Cho, H.; Jung, J. Distributed Strain Monitoring of Railway Composite Bogies Using a Brillouin Optical Correlation Domain Analysis System. Appl. Sci. 2018, 8, 1755. [CrossRef]

9. Minardo, A.; Coscetta, A.; Porcaro, G.; Giannetta, D.; Bernini, R.; Zeni, L. Distributed optical fiber sensors for integrated monitoring of railway infrastructures. Struct. Monit. Maint. 2014, 1, 173-182. [CrossRef]

10. Taiwan Central Weather Bureau. Observation Data of C0R170 Ping Tung Weather Station on the Date of 21 March 2017. Available online: https: / / e-service.cwb.gov.tw /HistoryDataQuery /DayDataController.do?command=viewMain\&station=C0R170 \&stname $=\% 25 \mathrm{E} 5 \% 25 \mathrm{~B} 1 \% 258 \mathrm{~F} \% 25 \mathrm{E} 6 \% 259 \mathrm{D} \% 25 \mathrm{~B} 1 \&$ datepicker=2017-03-21 (accessed on 1 October 2020) (In Traditional Chinese). 